\title{
Sexualidade para o homem em tratamento oncológico
}

\author{
Sexuality for men in oncological treatment \\ Sexualidad para el hombre en tratamiento oncológico
}

Itamara Barbosa Souza $^{1 *}$, Hulda Alves de Araújo Tenório², Everaldo de Lima Gomes Junior ${ }^{1}$, Isaac Carden Matias de Lima1', Raíssa Fernanda Evangelista Pires dos Santos² ${ }^{2}$ Luciana da Silva Viana².

\begin{abstract}
RESUMO
Objetivo: Compreender o significado de sexualidade para homens que estão em tratamento oncológico. Metodologia: Trata-se de uma pesquisa de campo, descritiva e de abordagem qualitativa, a coleta dos dados foi realizada por meio de uma entrevista com um roteiro semiestruturado. Para a análise dos dados foi utilizado à análise de conteúdo proposta por Bardin. Resultados: O câncer tem impacto na sexualidade dos sujeitos, pois diminui a produção do esperma, obriga o uso de camisinha e altera o estado físico geral. Quando a sexualidade foi abordada, a maioria dos entrevistados considerou-a como expressão do coito e do ato sexual, demonstrando decepção no que se refere a este aspecto. A ereção parece estar comprometida e os demais sentidos que conferem a sexualidade não são explorados pelos sujeitos que colocam suas forças na condição do tratamento. Conclusão: Sugere-se que a temática seja explorada por aqueles que cuidam diretamente de homens em tratamento oncológico, sendo este aspecto, mesmo que encarado como secundário, elucidado e encorajado. A sexualidade é uma necessidade e, portanto, parece ser importante para a qualidade e recuperação da vida.
\end{abstract}

Descritores: Sexualidade, Saúde do Homem, Terapêutica, Oncologia.

\begin{abstract}
Objective: To understand the meaning of sexuality for men who are on cancer treatment. Methodology: This is a field research, descriptive and qualitative approach, the data collection was performed through an interview with a semi-structured script. For the analysis of the data was used to the analysis of content proposed by Bardin. Results: Cancer has an impact on the subjects' sexuality, as it reduces the production of sperm, requires the use of a condom and changes the general physical state. When sexuality was approached, most of the interviewees considered it as an expression of sexual intercourse and sexual act, showing disappointment in this regard. Erection seems to be compromised and the other senses conferring sexuality are not exploited by the subjects who put their forces into the treatment condition. Conclusion: It is suggested that the thematic be explored by those who care directly for men in cancer treatment, and this aspect, even if viewed as secondary, elucidated and encouraged. Sexuality is a necessity and therefore seems to be important to the quality and recovery of life.
\end{abstract}

Keywords: Sexuality, Men's health, Therapy, Oncology.

\section{RESUMEN}

Objetivo: Comprender el significado de la sexualidad para los hombres que están en tratamiento oncológico. Metodología: Se trata de una investigación de campo, descriptiva y de abordaje cualitativo, la recolección de los datos fue realizada por medio de una entrevista con un guión semiestructurado. Para el análisis de los datos se utilizó el análisis de contenido propuesto por Bardin. Resultados: El cáncer tiene impacto en la sexualidad de los sujetos, pues disminuye la producción del esperma, obliga el uso de condón y altera el estado físico general. Cuando la sexualidad fue abordada, la mayoría de los entrevistados la consideró como expresión del coito y del acto sexual, demostrando decepción en lo que se refiere a este aspecto. La erección parece estar comprometida y los demás sentidos que confieren la sexualidad no son explotados por los

1 - Faculdade Estácio de Alagoas (FAL), Maceió - AL. * E-mail: itamara28pa@hotmail.com

2 - Universidade Federal de Alagoas (UFAL), Maceió - AL

SUBMETIDO EM: 10/2018

ACEITO EM: $11 / 2018$

PUBLICADO EM: 01/2019

REAS/EJCH | Vol. 11 (4) | e275 | DOI: https://doi.org/10.25248/reas.e275.2019 Página 1 de 9 
sujetos que ponen sus fuerzas en la condición del tratamiento. Conclusión: Se sugiere que la temática sea explotada por aquellos que cuidan directamente de hombres en tratamiento oncológico, siendo este aspecto, aunque parezca secundario, elucidado y alentado. La sexualidad es una necesidad y, por lo tanto, parece ser importante para la calidad y la recuperación de la vida.

Descriptores: Sexualidad, Salud del Hombre, Terapia, Oncología.

\section{INTRODUÇÃO}

O câncer é um termo que corresponde ao conjunto de mais de 100 enfermidades, e na atualidade é considerado um problema de saúde pública mundial (RODRIGUES e FERREIRA, 2011). Para o Instituto Nacional de Câncer (INCA) (2012), o câncer e outras doenças crônicas não transmissíveis (DCNT), vêm se tornando cada vez mais comuns no mundo e podem causar agravos devastadores para famílias inteiras. No Brasil, as estimativas para o ano de 2018 serão válidas também para o ano de 2019 e distingue a ocorrência de aproximadamente 600 mil casos novos de câncer para cada ano, reforçando a intensidade do problema do câncer no país.

É importante destacar que nas últimas décadas, os principais tipos de câncer com alto índice de morte foram: o câncer pulmonar (1,3 milhões), estômago (cerca de 1 milhão), fígado (662 mil), cólon (655 mil) e mama (502 mil) (INCA, 2012). No que se referem aos sujeitos comprometidos por tal patologia, segundo Modena et al. (2013) entre o público masculino, predominam os cânceres de pele não melanoma, próstata, pulmão, cólon e reto, e estômago.

A fisiopatologia do câncer é caraterizada pelo crescimento anormal das células, ou seja, a célula neoplásica sofre alteração nos seus mecanismos regulatórios de multiplicação e se torna independente de estímulos fisiológicos. Elas são classificadas como células malignas (MÜLLER et al., 2011). Sua evolução pode levar a proliferação destas células a outras regiões do corpo, caracterizando a metástase. Meohas et al. (2005), define metástase como, o resultado final de diversas fases interdependentes, um processo multifacetado que inclui uma complexa interação entre o tumor e organismo hospedeiro, uma sequência de acontecimentos com progressão do mesmo para diversas partes anatômicas do indivíduo.

Quando um paciente é diagnosticado com câncer são propostos alguns tratamentos, como cirurgia, radioterapia e quimioterapia, que causam possíveis reações adversas, as quais têm sido vivenciadas de forma negativa pela maioria dos pacientes (CUNHA et al., 2014).

O tratamento oncológico causa impacto na percepção da imagem corporal dos pacientes, mediante sua função cultural dentro da sociedade. Tendo em vista o processo de adoecimento e as medicações utilizadas, é comum que o homem sofra perda de peso, alopecia e perda da força muscular, interferindo em sua autoestima (TOFANI e VAZ, 2007; DÁZIO et al., 2009).

Moscheta e Santos (2012), ressaltam que um dos principais efeitos colaterais está relacionado aos aspectos da sexualidade, tendo destaque, não conseguir alcançar o clímax; impotência (incapacidade de ter ou de manter uma ereção); o cansaço referido durante o ato sexual, ou o não interesse pelo sexo devido à preocupação e a depressão.

É importante destacar que a impossibilidade do exercício da sexualidade gera nos homens com câncer a sensação de perda e/ou subtração da masculinidade, revelando que a identidade masculina se encontra intensamente pautada no exercício ativo e constante do ato sexual (TOFANI e VAZ, 2007; BARROS e MELO, 2009; DÁZIO et al., 2009). Segundo Teixeira et al. (2012), o ato genital/sexual, deixa de ser o único sentido da sexualidade, que na atualidade, abrange seu conceito como algo que envolve os aspectos singulares dos sentimentos e das sensações que envolvem o prazer. 
De acordo com Carraca et al. (2009), o desenvolvimento de ações voltadas para este público ainda se mostra como um grande desafio para gestores e trabalhadores de saúde, revelando a necessidade de maior compreensão dos aspectos socioculturais que permeiam as ações de prevenção, diagnóstico precoce e tratamento oncológico pela população masculina. Mesmo com o risco de desenvolvimento de doenças crônicas e graves, como o câncer, os homens não procuram ativamente a atenção primária, o que leva a busca tardia ao atendimento, favorecendo a morbidade.

Assim, para que haja uma assistência de qualidade ao paciente oncológico, os profissionais de saúde, em específico o enfermeiro, por atuar em todos os níveis de atenção, precisam ter conhecimentos técnicocientíficos e habilidades no relacionamento interpessoal. Uma vez que as ações de enfermagem necessitam serem integrais, participativas e resolutivas (GOMES et al., 2012).

O presente estudo justificou-se, pois se propôs incorporar o sujeito masculino nas temáticas abordadas dentro do contexto da saúde, bem como revelou um maior esclarecimento em relação à sexualidade, e como ela é vivenciada por pacientes em tratamento oncológico.

Cabe ressaltar que para o enfrentamento do câncer, são necessárias ações holísticas, integrais e humanizadas. Nesse contexto, o profissional de saúde deverá ser conhecedor do significado da sexualidade vivida pelos pacientes e estimula-la ao ponto de que tais indivíduos possam suprir suas necessidades sentindo-se mais vivos (GOMES et al., 2012).

Neste estudo traçou-se como objetivo conhecer o significado da sexualidade para homens que estão em tratamento oncológico, e identificar quais fatores estão interferindo na expressão da sexualidade destes indivíduos, além de compreender a importância deste assunto para estes.

\section{MÉTODOS}

Trata-se de estudo de campo, descritivo e de abordagem qualitativa. A pesquisa foi realizada Hospital Universitário Professor Alberto Antunes - Centro de Alta Complexidade em Oncologia - CACON/UFAL, localizado na Avenida Lourival Melo Mota, S/N, Tabuleiro do Martins - CEP: 57072-900 Maceió - Alagoas. Para a composição da amostra os critérios de inclusão foram: pacientes do sexo masculino, maiores de 18 anos, que estejam em tratamento oncológico (radioterapia e quimioterapia) há mais de 3 (três) meses na unidade CACON e em condições de responder a entrevista. $E$ os critérios de exclusão foram: pacientes provenientes de outras instituições para tratamentos provisórios na unidade; pacientes que fazem tratamento de outra patologia que não o câncer; e pacientes muito debilitados sem possibilidades físicas e cognitivas de responder as perguntas.

Primeiramente foram encaminhados um ofício e a carta de apresentação, ao responsável pela instituição explicando o projeto e esclarecendo objetivos, importância, e as possíveis repercussões advindas da pesquisa, bem como solicitado uma autorização para realização da pesquisa. Após aprovação do Diretor, o projeto foi encaminhado ao Comitê de Ética e Pesquisa (CEP) da Faculdade Estácio de Alagoas, através da Plataforma Brasil.

A pesquisa só teve início após a aprovação do CEP. Contudo, o único momento de entrada no campo de pesquisa foi para a obtenção de informações sobre o número de possíveis sujeitos que se enquadrariam nos critérios de exclusão e inclusão da pesquisa. O hospital disponibilizou estas informações sendo possível assim calcular o tamanho da amostra significativa para pesquisa, sendo esta uma exigência da unidade. Só no segundo momento, já após aprovação do CEP, é que os pacientes que se enquadraram nestes critérios, foram submetidos à entrevista com roteiro semiestruturado, onde anteriormente assinaram o Termo de Consentimento Livre e Esclarecido, bem como recebido sua via. 
Os sujeitos da pesquisa foram convidados a responder a entrevista com roteiro semiestruturado. Os horários e turnos para a coleta de dados foram definidos com a equipe médica e de enfermagem, visto conhecerem melhor a rotina do setor e o momento mais propício. As informações coletadas foram lançadas em planilha digital e, em sequência, foi realizada análise de conteúdo proposta por Bardin (2011) emergindo três categorias temáticas: $O$ paciente com câncer e sua condição de saúde; Entendimentos sobre a sexualidade e a sexualidade diante do tratamento oncológico.

Foram levados em consideração todos os aspectos éticos de pesquisas envolvendo seres humanos com protocolo aprovado sob registro de CAEE de oㅜ 58589916.0.0000.5012, sendo apreciado através do Comitê de Ética da Faculdade Estácio de Alagoas.

\section{RESULTADOS E DISCUSSÃO}

Os 13 participantes tinham entre 31 e 71 anos, todos são do sexo masculino com diagnóstico de câncer e em tratamento. Visando garantir $o$ anonimato dos entrevistados, seus nomes foram substituídos pela letra $P$ indicando "participador", seguida do respectivo número de identificação: P1, P2, P3, etc. Abaixo, será exposto o perfil dos entrevistados. Na Tabela 1 foram identificadas as características gerais dos participantes, como idade, estado civil, raça, escolaridade, procedência e comorbidades.

Tabela 1- Perfil dos homens com câncer em tratamento no Centro de Alta Complexidade em Oncologia CACON/UFAL, 2016.

\begin{tabular}{lllllll}
\hline Entrevistado & Idade & $\begin{array}{l}\text { Estado } \\
\text { Civil }\end{array}$ & Raça & Escolaridade & Procedência & Comorbidades \\
\hline P1 & 70 & Casado & Pardo & $1^{\circ}$ Grau incompleto & Urbano & Não \\
P2 & 53 & Casado & Pardo & $2^{\circ}$ Grau incompleto & Urbano & Não \\
P3 & 42 & Casado & Pardo & $2^{\circ}$ Grau Completo & Urbano & Não \\
P4 & 54 & Casado & Pardo & $2^{\circ}$ Grau incompleto & Urbano & Não \\
P5 & 31 & Casado & Pardo & $2^{\circ}$ Grau Completo & Urbano & Não \\
P6 & 66 & Casado & Pardo & $1^{\circ}$ Grau Incompleto & Urbano & Sim (HAS) \\
P7 & 71 & Casado & Pardo & Sem escolaridade & Urbano & Não \\
P8 & 63 & Casado & Pardo & $1^{\circ}$ Grau Completo & Urbano & Não \\
P9 & 69 & Casado & Pardo & $2^{\circ}$ Grau Completo & Rural & Não \\
P10 & 70 & Casado & Pardo & $1^{\circ}$ Grau incompleto & Rural & Sim (HAS) \\
P11 & 70 & Viúvo & Pardo & $2^{\circ}$ Grau completo & Urbano & Não \\
P12 & 69 & Casado & Pardo & $1^{\circ}$ Grau incompleto & Urbano & Sim (HAS e DM) \\
P13 & 42 & Casado & Pardo & $1^{\circ}$ Grau incompleto & Urbano & Não \\
\hline
\end{tabular}

Legenda: HAS- Hipertensão arterial; DM- Diabetes.

Fonte: dados da pesquisa, 2016.

$\mathrm{Na}$ caracterização dos participantes do estudo verificou-se que $62 \%$ dos homens apresentavam idade entre a faixa etária dos 61 e 71 anos. No que concerne ao estado civil é importante destacar que $92 \%$ afirmaram serem casados, $38 \%$ não concluíram o primeiro grau (ensino fundamental), $85 \%$ residem em área urbana e 77\% declaram não terem comorbidades (Hipertensão e Diabetes).

Em relação a nossa pesquisa, além da idade, que foi relatado como um fator que interfere na sexualidade do homem ao longo dos anos, a vivência da sexualidade durante o tratamento oncológico foi influenciada, especialmente por dois fatores: a realização de terapêuticas prévias e a sentimentos negativos atribuídos a prática sexual durante a terapêutica. 
Baseado no universo dos sujeitos e seguindo a proposta metodológica, apresentam-se, a seguir, os resultados e a análise do material. Os discursos dos participantes foram agrupados por temas abordados, que correspondem, basicamente, aos objetivos específicos da pesquisa. Em todos os discursos dos participantes, foi associada à ideia central correspondente para que, fosse possível analisar os depoimentos, tendo por referência a literatura científica atual sobre a temática em estudo.

\section{O paciente com câncer e sua condição de saúde}

A condição de saúde do paciente oncológico é uma preocupação frequente e de grande interesse, pois o aparecimento de uma enfermidade crônica como o câncer pode alterar sua condição geral, comprometendo a execução de atividades diárias e suas relações familiares (FREIRE et al., 2014). Contudo, após o início do tratamento e, consequentemente, redução de algumas características externas expostas pela doença, os indivíduos parecem acreditar que existe possibilidade de cura remetendo alegria e confiança através de discursos positivos e esperançosos, conforme falas abaixo:

P2: "[...] eu posso dizer que a minha situação geral de saúde é boa né! É porque o que estou vivendo não tenho levado em conta, mas estou crendo que em breve estarei curado" (Ideia central - boa condição de saúde).

P3: "[...] Eu uso colostomia, e assim fui pego de surpresa no tratamento é meio complicado, a gente que tinha $100 \%$ de saúde e gostava de desfrutar a vida. E de repente se passando por isso, mas Deus está no controle de tudo" (Ideia central - regular estado de saúde, mas existe esperança).

Algumas terapêuticas utilizadas no tratamento oncológico podem trazer características peculiares com alterações em várias funções do corpo, sendo as mais frequentes a queda de cabelo, ansiedade, náuseas, vômitos, anemia, fadiga, alterações renais e digestivas. Estes efeitos variam entre os pacientes, de acordo com sua função metabólica e da combinação das drogas utilizadas. Embora as maiorias destes efeitos desapareçam, assim que a terapêutica chega ao fim, durante este período, o indivíduo apresenta comprometimento de sua condição de vida (FIGUIREDO e SCHRAIBER, 2011).

Um procedimento comum utilizado como medida terapêutica ao paciente oncológico é a colostomia. De acordo com Pinto (2012), quando os indivíduos são submetidos à utilização de colostomia, logo se deparam com uma situação diferente da vivida no seu habitual, gerando um conflito com o seu eu. Devido ao novo conduto destinado a eliminações fisiológicas, surgem alterações físicas e emocionais levando-os a um desequilíbrio emocional, sendo necessário certo tempo para acontecer uma readaptação.

Embora, existam complicações no tratamento quimioterápico, radioterápico e cirúrgico, a esperança de cura oferecida por estas condutas, é capaz de promover reforço ao papel de encarar todos os efeitos contrários. Estas terapias são decisivas na diminuição da probabilidade de morte e no aumento do prognóstico de vida destes pacientes (COSTA e COELHO, 2011).

\section{Entendimentos sobre a sexualidade}

Fundamentadas em um saber empírico, os homens manifestam um sentimento de valorização em relação à sexualidade, baseado em uma responsabilidade cultural de masculinidade e procriação, e utilizam o autocuidado e a promoção da saúde como método de manutenção desta sexualidade ativa. $O$ termo "sexualidade" é vastamente conceituado e engloba inúmeros significados, dificilmente encaixando-se em uma definição única, podendo ser entendido como uma necessidade basal, um aspecto do ser humano que não pode ser separado de outros aspectos da vida, não sendo apenas sinônimo de coito (relação sexual) e nem se restringir ao orgasmo, mas parece ter relação com a energia que causa o contato e a intimidade, sendo expressa na forma de sentir, nos movimentos das pessoas, e como compartilham as trocas (FLEURY et al., 2011; TEIXEIRA at al., 2013; COSTA e COELHO, 2011). 
P2: "[...] Em relação à sexualidade creio que a sexualidade é você ter amor entre duas pessoas, não é só sexo, mas abrange verdadeiramente amor, o sentimento pelo outro, isso é o que é sobre sexualidade" (Ideia central - a sexualidade e sua subjetividade)

P13: "[...] Houve antes de fazer a cirurgia, eu tinha relação sexual normal, todos os dias e a hora que queria. Antes podia ter relação sexual duas ou três vezes na manhã era normal" (ideia central - Sexualidade sinônimo da ereção masculina).

A sexualidade em homens com câncer é uma extensão que pode ser comprometida ao longo de toda a vivência do adoecer. Manifestações físicas e emocionais são subsídios que colaboram para o surgimento de desordens sexuais. Em pacientes com neoplasias em órgãos ligados à sexualidade, como o câncer de próstata, essas manifestações podem ser ainda mais exacerbadas (FIGUIREDO e SCHRAIBER, 2011).

Nas falas de alguns pacientes existe uma necessidade de afirmação da masculinidade por meio da ereção, mesmo que a prática sexual não seja mais presente no relacionamento do casal. Entende-se que o pênis está associado à simbologia do poder e do domínio sobre a mulher, principalmente para o homem ocidental, ele é analisado como símbolo máximo da masculinidade e o atestado da sua virilidade. Deste modo, os pacientes oncológicos do sexo masculino têm dificuldades em se reconhecerem enquanto homens, quando se deparam com a dificuldade de manter uma relação com ereção e a penetração (VERENHITACH et al., 2014).

No entanto, um trabalho realizado por Giami et al. (2009) ressalta que as terapêuticas farmacológicas da impotência sexual masculina e de todos os problemas relacionados com a sexualidade, mostraram ser eficazes, bem tolerados e de simples utilização para ajudarem na resolução dos problemas comuns da vida habitual, colocando-se efetivamente em questão para ser utilizados durante tratamento oncológico.

Gonçalves et al. (2008) afirmam que ter aspectos da sexualidade presentes na vida é fundamental para a qualidade dela, contudo, tais aspectos poderão ser expressivamente comprometidos por uma patologia tão complexa como o câncer e suas diferentes terapêuticas, necessitando assim, de um olhar direcionado a temática.

\section{A sexualidade e o tratamento oncológico}

O tratamento oncológico é uma medida terapêutica fundamental para melhoria da condição de saúde, relacionada aos comprometimentos fisiológicos causados pela neoplasia. O uso da quimioterapia, radioterapia, cirurgias e outras medidas, são necessárias para melhorar o prognóstico de indivíduos com câncer reduzindo a condição patológica, e em alguns casos, até trazendo a cura. Porém, devido à agressividade com que estes medicamentos agem no organismo, parece comprometer a condição geral influenciando na homeostase e no funcionamento de sistemas do corpo humano, dentre eles, órgãos que atuam na práxis da sexualidade (MODENA et al., 2013; MODENA et al.,2014; MÜLLER et al., 2011; PORTO, 2003; VIEIRA et al., 2012).

Os depoimentos mostraram que a vivência da sexualidade no período de quimioterapia sofreu interferência direta das disfunções sexuais. Na verdade, pelo estigma da doença, muitos homens já chegam à quimioterapia, fisicamente e psicologicamente deprimidos e isso se agrava devido ao tratamento e seus efeitos colaterais. Contudo, a vivência da terapêutica e a busca pela cura passam a ser o foco principal.

P3: "[...] Hoje a gente só pode ter relação sexual com camisinha devido ao tratamento. Assim não dar para ficar à vontade. Só Deus para dar forças, é complicado." (Ideia central Consequências do tratamento).

P12: "[...] A gente se dar bem, porém meu relacionamento com minha esposa tá complicado, pois eu não tenho espermatozoide. Não, tá igual como era antes" (Ideia central - alterações funcionais do órgão sexual). 
A diminuição do espermatozoide foi uma queixa relatada na narrativa de P12. A incidência da disfunção do espermatozoide após a quimioterapia varia de estudo para estudo e depende de alguns fatores, como idade do paciente, função erétil prévia, comorbidades associadas e tipo de cirurgia empregada (FLEURY et al., 2011). Contudo, entende-se que tal característica parece deixar os pacientes desapontados e abatidos emocionalmente, pois interfere em sua atividade sexual.

Além de aspectos relativos à quantidade de esperma durante o coito, outra limitação apontada durante tratamento quimioterápico é o uso de camisinha até 48 horas após cada ciclo. Após receber quimioterapia, o indivíduo apresenta doses elevadas de agente tóxico podendo estar presente inclusive nas gônadas masculinas, sendo necessária proteção da parceira para evitar contato químico (CAMPOS et al., 2011).

\section{$O$ câncer e as relações interpessoais}

A qualidade dos relacionamentos também é um meio terapêutico capaz de favorecer e intensificar a capacidade vital dos indivíduos oncológicos. A compreensão e o sentimento de cumplicidade são valores significativos para recuperação e enfrentamento de pessoas com câncer às adversidades oriundas da doença e do tratamento. Muitas vezes, estas relações estão comprometidas, e um sentimento de incompetência e desesperança, por parte do paciente em tratamento, agrava o quadro físico e psicológico. É importante destacar que o apoio de entes queridos e de pessoas próximas, pode promover uma melhor recuperação destes pacientes e um sentimento de valoração compatível com as necessidades daqueles que percorrem um caminho doloroso (RODRIGUES e FERREIRA, 2011).

P8: "[...] não tenho vida sexual ativa depois da cirurgia. Na vida de casado, não interfere em nada. A minha esposa respeita a nossa relação e estar conformada, até porque ela já teve câncer de mama há 8 anos atrás e entende. A gente procura se ajeitar, tem que ficar bom pros dois. $O$ nosso relacionamento continua do mesmo jeito, apesar das dificuldades" (Ideia central Compreensão mútua).

Gomes et al. (2011), destacam que a população masculina precisa de um acolhimento ancorado na comunicação como aquele que se fundamenta na conversa e da escuta mútua. Essa comunicação pode ser estruturante num acolhimento em que são envolvidos sujeitos pertencentes a diversos contextos.

A narrativa mostrara que o aparecimento de uma enfermidade crônica como o câncer pode ocasionar alterações para o relacionamento do homem e sua parceira e neste contexto, a comunicação estabelecida entre o casal tem um papel expressivo no casamento, revelando-se como fortalecedora da relação (LINDAU et al., 2011).

Além disso, ressalta-se que o relacionamento afetivo e sexual pode sofrer interferência do desconhecimento sobre os aspectos relativos ao tratamento e suas alterações fisiológicas. Esse desconhecimento colabora para o surgimento de crenças que comprometem a sexualidade, provocam desconfianças e o afastamento do casal (ABREU et al., 2013).

Ao enfermeiro que proporciona uma assistência a esse homem, é necessário estar à disposição para ouvir suas ansiedades, elucidar suas dúvidas, expor à temática, envolver a parceira, encorajar uma atitude mais humana que englobe aspectos da sexualidade no dia-a-dia destes indivíduos, visto a sexualidade ser uma área essencial da qualidade de vida de qualquer pessoa (MERCADANTE et al., 2010).

\section{CONCLUSÃO}

O câncer pode ocasionar alterações nos diferentes aspectos biopsicossociais, tendo impacto na sexualidade dos sujeitos. O uso de colostomia, a diminuição do esperma, o uso de camisinha e as alterações do estado físico geral são fatores que debilitam emocionalmente os homens, pois alteram aspectos físicos e comportamentais vivenciados durante toda uma vida, limitando-os em sua condição de dono de casa, 
funcionário e marido. Quando a sexualidade foi abordada, a maioria dos entrevistados considerou-a como expressão do coito e do ato sexual, demonstrando decepção no que se refere a este aspecto. A ereção pareceu estar comprometida e os demais sentidos que conferem a sexualidade não são explorados pelos sujeitos. A comunicação e o apoio da parceira na procura por adaptações a nova condição de vida e consequentemente da sexualidade, mostrou-se positiva, embora apenas um dos sujeitos tenha relatado tal fato. Diante disso sugere-se que a temática seja explorada por aqueles que cuidam diretamente desses indivíduos, sendo este aspecto, mesmo que encarado como secundário, elucidado e encorajado. Aspectos voltados à sexualidade é uma necessidade e, portanto, é ser importante para qualidade e recuperação da vida.

\section{REFERÊNCIAS}

1. ABREU AS, CRUZ ACA, CORTEZ EA, et al. Estratégias para a prevenção do câncer de próstata. Revista de Pesquisa Cuidado Fundamental, Rio de Janeiro, 2013; 5(2): 3795-07.

2. BAINE M, SAHAK FLC, et al. Marital status and survival in pancreatic cancer patients: A SEER bsed analysis. Plos One, 2011; 6(6): 1-9.

3. BARDIN L. Análise de conteúdo. 70. ed. São Paulo; 2011.

4. BARROS EM, MELO MCB. Câncer de pênis: perfil sócio-demográfico e respostas emocionais à penectomia em pacientes atendidos no Serviço de Psicologia do Hospital de Câncer de Pernambuco. Revista SBPH, 2009; 12(1): 99-111.

5. BRASIL. Instituto Nacional de Câncer. ABC do câncer: abordagens para o controle do câncer Rio de Janeiro, 2011.

6. BERTOLODO AS, PASQUINI VZ. Câncer de próstata: um desafio para a saúde do homem. Revista de Enfermagem UNISA, São Paulo, 2010; 11(2): 138-142.

7. CAMPOS HLM, DIAS FMV, MORAES SC, et al. Aspectos culturais que envolvem o paciente com diagnóstico de neoplasia de próstata: um estudo na comunidade. Revista Brasileira de Cancerologia, Rio de Janeiro, 2011; 57(4): 439-501.

8. CARRARA S, RUSSO JA, FARO L. A política de atenção à saúde do homem no Brasil: os paradoxos da medicalização do corpo masculino. Physis Revista de Saúde Coletiva, Rio de Janeiro, 2009; 19(3): 659-678.

9. COSTA LHR, COELHO ECA. Enfermagem e sexualidade: revisão integrativa de artigos publicados na Revista Latino-Americana de Enfermagem e na Revista Brasileira de Enfermagem. Rev. Latino-Am. Enfermagem, São Paulo, 2011; 19(3): 1-10.

10. CUNHA NF, ANJOS ACY, GONÇALVES MM, et al. Consulta multiprofissional à mulher com câncer de mama em quimioterapia: humanização da assistência. Revista de Enfermagem UFPE, 2014; 8(2): 484-488.

11. DÁZIO EMR, SONOBE HM, ZAGO MMF. Os sentidos de ser homem com estoma intestinal por câncer colorretal: uma abordagem na antropologia das masculinidades. Revista Latino-Americada de Enfermagem, São Paulo, $2009 ; 17(5)$.

12. FIGUIREDO WS, SCHRAIBER LB. Concepções de gênero de homens usuários e profissionais de saúde de atenção primaria e os possíveis impactos na saúde da população masculina. Revista Ciência Saúde coletiva, Rio de Janeiro, 2011; 16(1): 955-944.

13. FLEURY HJ, PANTAROLO HS, ABDO CHN. Sexualidade em Oncologia. Diagnóstico e Tratamento, São Paulo, 2011; 16(2): 86-90.

14. FREIRE MEM, SAWADA NO, FRANÇA ISX, et al. Qualidade de vida relacionada à saúde de pacientes com câncer avançado: uma revisão integrativa. Revista da Escola de Enfermagem da USP, São Paulo, 2014; 48(2): 357-367.

15. GODOY AS. Introdução à pesquisa qualitativa e suas possibilidades. Revista Adm. Emp. São Paulo, 1995; 35(2):57-63.

16. GOMES R, NASCIMENTO EF, ARAÚJO FC. Por que os homens buscam menos os serviços de saúde do que as mulheres? As explicações de homens com baixa escolaridade e homens com ensino superior. Caderno Saúde Pública, 2007; 23(3): 565-574.

17. GOMES LMX, BARBOSA AO, GUIMARÃES IR, et al. Assistência de enfermagem ao paciente oncológico adulto: uma revisão integrativa. Revista Digital, Buenos Aires, 2012; 16(164).

18. GOMES R; REBELLO LEFS, NASCIMENTO EF. et al. Atenção Básica à Saúde do homem sob a ótica do usuário: um estudo qualitativo em três serviços do Rio de Janeiro. Revista Ciência e Saúde Coletiva, Rio de Janeiro, 2011; 16(11): 4513-4521. 
19. GONÇALVES IR, PADOVANI C, POPIM RC. Caracterização epidemiológica e demográfica do câncer de próstata. Ciência \& Saúde Coletiva, Rio de Janeiro, 2008; 13(4): 1337-1342.

20. Instituto Nacional de Câncer, Ministério da Saúde: Incidência do Câncer no Brasil. Rio de Janeiro, 2018.

21. LAKATOS EM, MARCONI MA. Técnica de pesquisa. 3 ed. São Paulo: Altas, 1996.

22. LINDAU S, SURAWSKA H, BARON S. Communication about sexuality and intimacy in couples affected by lung cancer and their clinical care providers. Psycho-Oncology, 2011; 20(2): 179-85.

23. MEOHAS W, PROBSTNER D, VASCONCELLOS RAT, et al. Metástase óssea: revisão da literatura. Revista Brasileira de Cancerologia, 2005; 5(1): 43-47.

24. MERCADANTE S, VITRANO V, CATANIA V. Sexual issues in early and late stage cancer: a review. Support Care Cancer, 2010; 18(6): 659-65.

25. MINZINI EJ. Entrevista semi-estruturada: análise de objetivos e de roteiros. In: Seminário Internacional sobre pesquisa e estudos qualitativos. 2, 2004, Bauru. A pesquisa qualitativa em debate. Anais. Bauru: USC, 2004. CD-ROOM.

26. MODENA CM, MARTINS AM, RIBEIRO RBN, et al. Os homens e o adoecimento por câncer: um olhar sobre a produção científica brasileira. Revista Baiana de Saúde Pública, Bahia, 2013; 37(3): 644-660.

27. MODENA CM, MARTINS AM, GAZZINELLI AP, et al. Câncer e masculinidades: sentidos atribuídos ao adoecimento e ao tratamento oncológico. Temas psicol. 2014; 22(1): 67-78.

28. MOSCHETA MS, SANTOS MA. Grupos de apoio para homens com câncer de próstata: revisão integrativa da literatura. Revista Ciências \& Saúde Coletiva, 2012; 17(5): 1225-1233.

29. MÜLLER AM, SCORTEGAGNA D, MOUSSALLE LD. Paciente Oncológico em Fase Terminal: Uma Abordagem do Fisioterapeuta. Revista Brasileira de Cancerologia, 2011; 57(2): 207-215.

30. PORTO JB. A sexualidade em portadores de câncer - os médicos e o que pode ser feito. Revista Brasileira de Sexualidade Humana, 2003; 14(2):229-230.

31. RODRIGUES JSM, FERREIRA NMLA. A experiência da família no cuidado domiciliário ao doente com câncer: uma revisão integrativa. Revista Eletrônica de Enfermagem, 2011; 13(2): 338-346.

32. SABO D. Comprender la salud de los hombres: um enfoque relacional y sensible al género. Organización panamericana de la Salud, 2000.

33. VERGARA SC. Projetos e relatórios de pesquisa em administração. 5. ed. São Paulo: Atlas, 2004.

34. VIEIRA CG, ARAÚJO WS, VARGAS DRM. O homem e o câncer de próstata: prováveis reações diante de um possível diagnóstico. Revista Científica do ITPAC, 2012; 5(1).

35. TEIXEIRA MM, ROSA RP, SILVA SN, et al. O enfermeiro frente à sexualidade na terceira idade. Revista da Universidade Ibirapuera, São Paulo, 2013; 3: 50-53.

36. TOFANI ACA, VAZ CE. Câncer de Próstata, sentimento de importância e fracassos ante os cartões IV e VI do Rorschach. Revista Interamericana de Psicologia, 2007; 41(2): 197-204.

37. VERENHITACH BD, MEDEIROS JN, ELIAS S, et al. Câncer de mama e seus efeitos sobre a sexualidade: uma revisão sistemática sobre abordagem e tratamento. Revista FEMINA, 2014; 42(1): 4-10. 